\title{
Occult HCV Infection (OCI) Diagnosis in Cirrhotic and Non-cirrhotic Naïve Patients by Intra-PBMC Nested Viral RNA PCR
}

\author{
Mohamed Darwish Ahmed Abd Alla*1, Saleh Ahmed Elibiary ${ }^{1}$, George Y. Wu ${ }^{2}$ \\ and Mostafa Kamel El-Awady ${ }^{3}$ \\ ${ }^{1}$ Tropical Medicine Department, Faculty of Medicine, Al-Azhar University, Cairo, Egypt; ${ }^{2}$ Department of Medicine, Division of \\ Gastroenterology-Hepatology, University of Connecticut Health Center, Farmington, CT, USA; ${ }^{3}$ Department of Microbial \\ Biotechnology, National Research Center, Cairo, Egypt
}

\begin{abstract}
Background and Aims: Occult HCV infections (OCIs) include IgG antibody seronegative cryptogenic (COCIs), as well as seropositive secondary naïve (SNOCIs) and experienced (SEOCIs) cases. We used peripheral-blood-mononuclear-cell (PBMC)-PCR to evaluate COCIs and SNOCIs prevalence, serum HCV spontaneous disappearance (SCSD) in naïve cirrhotics and non-cirrhotics, intra-PBMC HCV-RNA strands in relation to cirrhosis density in naïve non-viremia cases, and HCV-RNA seroconversion after 1 year of solitary naïve intra-PBMC infection. Methods: The anti-HCV IgG antibodypositive naïve-patients $(n=785)$ were classified into viremic $(n=673)$ and non-viremic $[n=112$, including non-cirrhotics $(n=55)$ and cirrhotics $(n=57)]$, and 62 controls without evidence of HCV-infection. Controls and post-HCV non-viremia cases $(n=62+112=174)$ were submitted to hepatic FibroscanElastography evaluation. All subjects $(n=847)$ were screened for intra-PBMC HCV-RNA sense and antisense strands by nested-PCR. Results: Naïve-OCI cases (4.84\%) that were diagnosed by PBMC-PCR significantly raised the total numbers of HCV-infection to $714(p=0.01)$. The percent positivity of SNOCIs $(34.82 \%)$ was significantly higher than for asymptomatic-COCIs (3.125\%, $p=0.0001)$. Comparing PBMC-PCR with single-step-reverse-transcription (SRT)-PCR for identification of SCSD in naïve IgG antibody-positive nonviremia patients $(n=112)$ revealed a decline in SCSD prevalence by PBMC-PCR (from $14.27 \%$ to $9.3 \%$ ), regardless of presence of hepatic cirrhosis $(p=0.03)$. SCSD was found to be higher by PBMC-PCR in non-cirrhotics compared to cirrhotics $(p=0.0001)$, with an insignificant difference when using SRT-PCR $(p=0.45)$. Intra-PBMC HCV-RNA infection was significantly more frequent in cirrhotics compared to both non-cirrhotics and controls $(p<0.0005)$. An increased
\end{abstract}

Keywords: OCIs; PBMCs; Naïve; Cirrhosis.

Abbreviations: COCIs, cryptogenic occult HCV infection; DAA, direct-acting antiviral; HCC, hepatocellular carcinoma; HCV, hepatitis C virus; OCIs, occult HCV infections; PBMCs, peripheral blood mononuclear cells; RAVs, resistance-associated variants; SCSD, serum HCV spontaneous disappearance; SEOCIs, secondary experienced occult HCV infections; SNOCIs, secondary naïve occult HCV infection; SRT, single-step reverse transcription; SVR, sustained virologic response.

Received: 26 May 2017; Revised: 25 July 2017; Accepted: 11 August 2017

*Correspondence to: Mohamed Darwish Ahmed Abd Alla, El-Hussein University Hospital, Al-Azhar University, Gouhar Al-Kaed Street, Al-Darasah, Cairo 11675, Egypt. Tel: +20-109-417-5209, Fax: +20-25123091, E-mail: darwish0716@azhar.edu.eg hepatic fibrosis density was recognized in intra-PBMC HCVRNA infection with sense $(p=0.0001)$ or antisense strand $(p=0.003)$. HCV-RNA seroconversion was associated with intra-PBMC infection when both sense and antisense strands were detected $(p=0.047)$. Conclusions: Intracellular HCVRNA evaluation is crucial for diagnosing OCIs and addressing relapse probability.

Citation of this article: Abd Alla MDA, Elibiary SA, Wu GY, El-Awady MK. Occult HCV infection (OCI) diagnosis in cirrhotic and non-cirrhotic naïve patients by intra-PBMC nested viral RNA PCR. J Clin Transl Hepatol 2017;5(4):319-326. doi: 10.14218/JCTH.2017.00034.

\section{Introduction}

In 2004, Pham et al. ${ }^{1}$ reported occult HCV infections (OCIs) in anti-HCV IgG antibody-positive naïve patients who had spontaneous recovery after self-limited HCV-infections and in experienced cases with sustained virologic response (SVR) after interferon treatment. During the same year, Castillo et al. ${ }^{2}$ described HCV-RNA existence in anti-HCV IgG antibodynegative naïve patients who presented with active hepatocellular damage. Further refinement of the definitions of OCIs included: a) recognition of intracellular RNA strands in postinfection non-viremia naïve cases, which is termed spontaneous HCV disappearance (SCSD) and manifests as secondary naïve OCIs (SNOCIs); b) identification of post-treatment non-viremia cases, which have intracellular HCV-RNA strand infections, and manifest as secondary experienced OCIs (SEOCIs). ${ }^{3,4}$

Recently, baseline diagnosis and post-treatment follow-up of both SNOCIs and SEOCIs by peripheral blood mononuclear cell (PBMC) HCV-PCR in Egyptian populations addressed both validation of PBMC-PCR as a diagnostic test that can diagnose intracellular HCV when single-step reverse transcription (SRT)-PCR is negative and the association of intra-PBMC infections with liver cirrhosis in naïve non-viremia patients. The authors recommended eradication of intra-PBMC RNAstrands in SEOCIs to avoid HCV-RNA seroconversion and subsequent post-treatment relapse. ${ }^{5}$ A third category of OCIs is known as cryptogenic occult HCV-infection (COCIs), which is diagnosed in the IgG-seronegative population by detecting the intracellular RNA strands. Its prevalence is around $3.5 \%$ in asymptomatic populations, ${ }^{3}$ which might 
Abd Alla M.D.A. et al: OCI diagnosis in naïve patients by PBMC PCR

have active replication of HCV inside PBMCs, as identified by detection of either the viral NS3-protein or antisense-strand. ${ }^{6}$

In 1999, El-Awady et al. ${ }^{7}$ predicted that assay for viral replication in PBMCs enhances sensitivity of diagnosing and monitoring HCV associated hepatitis. Accordingly, intra-PBMC detection of HCV-RNA by PCR is currently used to diagnose OCIs in non-viremia patients. ${ }^{5}$ It is obvious that the immune system is not only a part of an OCI patient's reaction to the infection but that it also serves as a host to the viral RNA, thereby facilitating its replication, as indicated by antisense HCV-RNA strands found in the PBMCs of these patients. ${ }^{8}$ Keeping in mind that HCV-infection is an important and treatable cause of liver disease and the fact that patients with cryptogenic liver diseases can potentially develop cirrhosis and hepatocellular carcinoma, investigating the prevalence of $\mathrm{HCV}$ and developing more accurate techniques to diagnose OCIs in these patients are critically important. ${ }^{9}$

Some researchers ${ }^{10}$ have expressed doubts about using SRT-PCR in post-treatment follow-up. They have concluded that HCV cure in individuals achieving a state of undetectable viremia after an 8 to 12 -week course of direct-acting antiviral (DAA) therapy may not be entirely valid. The same researchers recommended careful longitudinal follow-up utilizing highly sensitive assays and unique approaches to viral detection. Because PBMCs present a convenient extrahepatic home for HCV adoption, translation, transcription, assembly and finally release into the serum and/or other adjacent cells, ${ }^{6}$ cellular PCR can be used to further refine the definitions of clinical presentations of spontaneous HCV clearance. Recognition of intra-PBMC HCV strands in $\mathrm{SNOCIs}^{5}$ should refer to regression of using the 'spontaneous $\mathrm{HCV}$-clearance' term. In populations of HCV endemic areas, the SCSD is estimated to be around $25 \% .{ }^{11}$ However, cellular HCV spontaneous disappearance has not been evaluated before the current report.

On the other hand, the post-treatment RNA genomic seroconversion has been attributed to either treatment failure or new infection, and is known as HCV-relapse, ${ }^{12}$ which has not been described in naïve subjects who present with spontaneous viral clearance because no temporal follow-up is recommended. Till now, naïve cirrhotic patients who present with nonviremia, but have evidence of hepatocellular damage and positive intra-PBMC HCV-RNA strand infection, have not been considered as candidates for DAA therapy. ${ }^{2}$ However, eradication of intra-PBMC HCV-infection is recommended in naïve cirrhotics and experienced post-treatment patients, respectively, because of association with cirrhotic changes and probability of relapse in more than $18 \% .^{5}$

Considering the high cure rate of DAA therapy, physicians are looking for effective treatments for COCIs and SNOCIs. Patients with OCIs should be afforded the option of getting rid of the intracellular HCV genomic materials, to prevent the subsequent hepatic fibrosis. ${ }^{9}$ For this reason, the current study was designed to shed more light on the OCI problem among a population of cases from one of the most endemic areas in the world for HCV-infection, in the hopes of promoting attention to this unmet challenge to satisfy clinical practice demands.

The specific aims of the current study included the use of PBMC-PCR in the evaluation of a) prevalence of asymptomatic COCIs and SNOCIs, b) SCSD in naïve cirrhotics and noncirrhotics, c) intra-PBMC HCV-RNA sense and antisense strand infections in relation to cirrhosis density in naïve non-viremia cases, and d) rate of HCV-RNA seroconversion after 1 year of solitary naïve intra-PBMC infection.
Methods

\section{Study subjects}

All study subjects ( $n=847$ ) were treatment-naïve and outpatient-visitors to the Infectious Disease Clinic. All subjects were screened by SRT-PCR between January 2015 and February 2017. Inclusion criteria were age between 18 and 70 years-old and positivity for serum anti-HCV IgG antibodies; controls were recruited from the population that was clinically and serologically free of HCV. Exclusion criteria included hepatocellular carcinoma (HCC) and Child C classification. Ethical committee approval for the study was obtained before patient enrollment (Registration No. 10231, National Research Center).

Sample size in each group depended upon availability of subjects that fulfill the inclusion criteria during the study period. Study subjects ( $n=847$ ) included the following subgroups: anti-HCV IgG antibody-positive naïve patients $(n=$ 785) who were classified into chronic HCV-viremic $(n=673)$ and post-HCV non-viremic cases $(n=112)$, with the last group further divided into non-cirrhotic $(n=55)$ and cirrhotic $(n=57)$ subgroups. Controls included 62 participants without evidence of HCV-infection. Controls and post-HCV nonviremia cases ( $n=174$ ) submitted to hepatic elastography evaluation by Fibroscan. All subjects $(n=847)$ were screened for presence of intra-PBMC HCV-RNA sense and antisense strands by nested-PCR.

\section{ELISA for HCV IgG antibody detection}

The procedure was performed as described. ${ }^{13}$ The thirdgeneration ELISA contained reconfigured NS3 and core antigens and in addition a newly incorporated antigen from the NS5 region. This assay was used as a preliminary screening test to enroll cases in the current study.

\section{Real-time PCR for quantification of HCV RNA}

The collection and transportation of specimens, RNA isolation, SRT-PCR procedure, internal control of the isolated RNA and/ or contamination, and quantification of the HCV PCR were all performed as described by Abd Alla and El-Awady ${ }^{5}$ in 2017.

\section{Amplification of intracellular HCV RNA genomes by strand-specific RT-PCR}

\section{Extraction of RNA from PBMCs}

Peripheral blood $(200 \mu \mathrm{L})$ was diluted with $10 \mathrm{~mL}$ freshly prepared red blood cell samples in alkaline buffer $(38.8 \mathrm{mmol} / \mathrm{L}$ $\mathrm{NH}_{4} \mathrm{Cl}, 2.5 \mathrm{mmol} / \mathrm{L} \mathrm{K}_{2} \mathrm{HCO}_{3}, 1 \mathrm{mmol} / \mathrm{L}$ EDTA; pH 8.0). After 10 min incubation at room temperature, nucleated cells were washed with the same buffer, the cells were dissolved in $500 \mu \mathrm{L}$ anti nuclease solution (4 mol/L guanidinium isothiocyanate containing $25 \mathrm{mmol} / \mathrm{L}$ sodium citrates, 0.5 sarcosyl and $0.1 \mathrm{~mol} / \mathrm{L}$ B-mercaptoethanol). A single-step method described by ${ }^{14}$ and modified by ${ }^{15,16}$ followed to carry out the RNA extraction.

\section{Retrotranscription PCR of sense and antisense strands of HCV RNA}

Detection of HCV RNA strands in PBMCs was performed as described by Lohr et al. ${ }^{17}$ The sequences of primers used in 
this study are: $1 \mathrm{CH}, 5^{\prime}$ ggt gca cgg tct acg aga cct ${ }^{\prime} 3^{\prime} ; 2 \mathrm{CH}, 5^{\prime}$ aac tca tgt ctt cac gca gaa3'; P2, 5'tgc tca tgg tgc acg gtc ta3'; P3, 5'ctt tcg cga ccc aac act ac3'; and P4, 5'aga gcc ata gtg gtc tgc gg $3^{\prime}$. The other steps for the retrotranscription PCR procedure for sense and antisense strands of HCV RNA were done as previously reported by Abd Alla and El Awady. ${ }^{5}$

\section{Elastography Fibroscan}

The procedure was carried out as previously described. ${ }^{18}$ Briefly, patients were placed in supine position, with their right arm in maximal abduction. All measurements were performed in the right lobe of the liver through the costal space. The tip of the probe transducer was covered with coupling gel and placed on the skin between the ribs at the right liver lobe level. The operator, assisted by ultrasound time motion and a mode images provided by the system, located a portion of the liver that was at least $6 \mathrm{~cm}$ thick and free of any large vascular structures. Once the area of measurement was located, the operator pressed the probe button to begin image acquisition. The measurement depth ranged from $25 \mathrm{~mm}$ to $45 \mathrm{~mm}$, and 10 valid measurements were obtained for each patient. The results were expressed in units of kilopascal $(\mathrm{kPa})$. All the non-viremic subjects underwent the examination with a normal prob. The success rate ${ }^{19}$ of the examination was calculated as the ratio between the number of measurements validated by the machine and the total number of attempted measurements. The liver stiffness corresponds to the median value of the validated measurements. The interquartile range (IQR) was used as the interval around the median that contained $50 \%$ of the valid measurements. To be considered interpretable and valid, the examination must have included at least 10 measurements, with a SR of at least $66 \%$; and, the IQR must not have exceeded $33 \%$ of the results of the examination.

\section{Statistical analysis}

The diagnostic procedure (PBMC-PCR) used was compared to the SRT-PCR (gold standard test) in a cross-sectional study that contained two groups of selected subjects. All cases that fit the inclusion criteria during 25 consecutive month period were included in the study. All tests performed were two-sided and statistical significance was considered at a $p$-value of 0.05 . SSPS version 9.0 for Windows (Chicago, Illinois, USA) was used in the data analysis. We compared the mean results by the Student's $t$-test (for variables with normal distribution) or by the Mann-Whitney $U$ test (for variables with non-normal distribution). Categorical variables were compared using the chi-square or Fisher's exact tests. To study the existence of correlation between the variables, Pearson's (for variables with Gaussian distribution) or Spearman's (for non-normally distributed variables) correlation coefficients were determined.

\section{Results}

\section{Advantages of combined screening with both SRT-PCR and PBMC-PCR}

As illustrated in Figure 1, both SRT-PCR and PBMC-PCR were used in screening of study populations with (Fig. 1A and $1 \mathrm{~B}$ ) and without (Fig. 1C) controls for HCV-infection. All cases which tested positive for HCV-infection by SRT-PCR ( $n=673 / 847,79.46 \%$; Fig. $1 \mathrm{~A} \& \mathrm{~B}$ and $673 / 785,85.733 \%$; Fig. 1C) were also positive for intra-PBMC HCV RNA strands. Patients who presented with non-viremia, as identified by SRT-PCR, consisted of anti-HCV IgG antibody negative controls $(n=62)$ and IgG antibody-positive naïve patients $(n=112)$.

Testing all RNA-seronegative subjects (controls $n=62+$ IgG-seropositive $n=112$ ) by cellular-PCR for intra-PBMC HCV-infection revealed 60/847 (7.1\%) of negative controls, $73 / 847(8.62 \%)$ of negative naïve post-HCV infection patients, and $41(4.84 \%)$ patients positive for intra-PBMC RNA strands, as demonstrated in Figure 1B. Adding the extra positive patients $(n=41)$ who presented with solitary intra-PBMC HCV-RNA strand infection to the baseline RNAseropositive patients $(n=673 / 847,79.46 \%)$ significantly raised the overall percent positive patients for HCV-infection

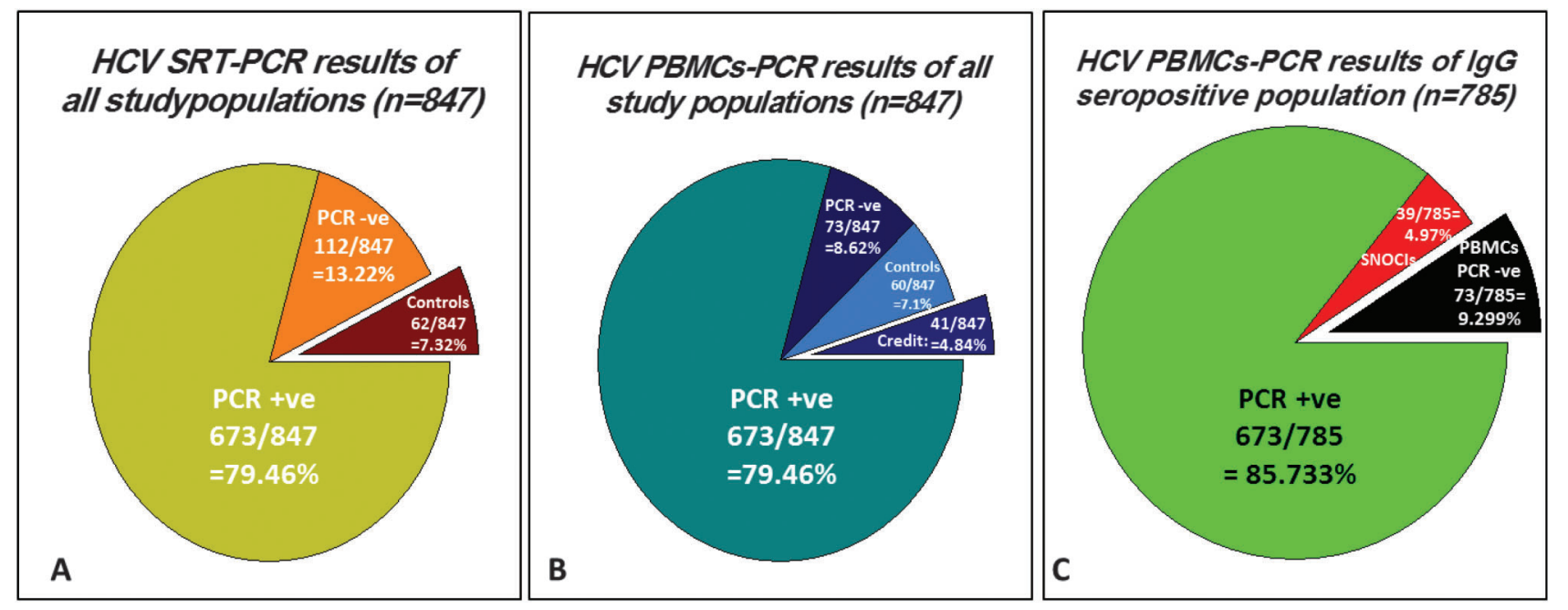

Fig. 1. Advantages of combined serum and PBMCs (B and C) screening over solitary serum screening for HCV infection (A) by PCR. PBMC testing of nonviremic subjects detected an extra $41(4.84 \%)$ and $39(4.97 \%)$ infections upon adding (B) or subtracting (C) controls, respectively. The higher numbers of HCV diagnosis obtained by detecting intracellular RNA strands significantly raised the overall frequencies of HCV diagnosis by PBMCs compared to SRT-PCR ( $p=0.011$ for panel B and $p=0.0029$ for panel C). As noted in panel C, the frequency of SNOCIs $(39 / 785,4.968 \%)$ is significantly lower than the frequency among patients with negative PCR $(73 / 785$, $9.3 \%)(p=0.001)$. 
Table 1. HCV RNA genomic detection within PBMCs compared to serum in naïve post-HCV infection

\begin{tabular}{lll}
\hline $\begin{array}{l}\text { Type of HCV } \\
\text { genomic PCR }(n)\end{array}$ & $\begin{array}{l}\text { HCV RNA- } \\
\text { negative, } n(\%)\end{array}$ & $\begin{array}{l}\text { HCV RNA- } \\
\text { positive, } n(\%)\end{array}$ \\
\hline SRT-PCR (785) & $112(14.27)$ & $673(85.73)$ \\
PBMC-PCR (785) & $73(9.30)$ & $712(90.70)$ \\
$\begin{array}{l}\text { Fisher's exact } \\
\text { 2-tailed } p\end{array}$ & 0.00285545 & \\
\hline
\end{tabular}

HCV diagnosis by intra-PBMCs RNA genomic detection was significantly highe compared to SRT-PCR ( $p=0.0029)$. PBMC-PCR-based frequency of negative HCV infection among naïve patients $(9.31 \%)$ was significantly lower than the SRT-PCRbased frequency $(14.16 \% ; p<0.00001)$.

Table 2. COCIs and SNOCIs in anti-HCV IgG antibody-positive and RNA seronegative naïve patients compared to negative controls

\begin{tabular}{llll}
\hline $\begin{array}{l}\text { Studied group } \\
(n)\end{array}$ & $\begin{array}{l}\text { COCIs, } \\
n(\%)\end{array}$ & $\begin{array}{l}\text { SNOCIs, } \\
n(\%)\end{array}$ & $\begin{array}{l}\text { Non-OCIs, } \\
n(\%)\end{array}$ \\
\hline $\begin{array}{l}\text { Negative } \\
\text { controls (62) }\end{array}$ & $2(3.125)$ & $0.0(0.0)$ & $60(96.875)$ \\
$\begin{array}{l}\text { IgG-positive/ } \\
\begin{array}{l}\text { SRT-PCR- } \\
\text { negative (112) }\end{array}\end{array}$ & $0.0(0.0)$ & $39(34.82)$ & $73(65.18)$ \\
$\begin{array}{l}\text { Fisher's exact } \\
\text { 2-tailed } p\end{array}$ & 0.00000025 & & \\
\hline
\end{tabular}

As diagnosed by PBMC-PCR, the prevalence of SNOCIs in anti-HCV IgG antibodypositive patients $(34.82 \%)$ is highly significant compared to the prevalence of COCIs $(3.1 \%)$ in negative controls $(p<0.00001)$.

( $n=714 / 847,84.3 \% ; p=0.012$ ). Figure $1 \mathrm{C}$ shows that prevalence of SNOCIs in the HCV IgG antibody-seropositive population $(39 / 785,4.968 \%)$ was significantly lower than the frequency of RNA-seronegative PBMC-PCR (73/785, 9.3\%; $p=0.001$ ).

Table 1 demonstrates results of both SRT-PCR and PBMC-PCR regarding evaluation of naïve post-HCV RNAseronegative and naïve RNA-seropositive infections without negative controls. Diagnosis of intra-PBMC RNA strand infection ( $n=712 / 785,90.7 \%)$ was significantly higher than that of SRT-PCR $(673 / 785,85.73 \% ; p=0.0029)$. The number of HCV infection-free cases diagnosed by SRT-PCR (112/785, $14.27 \%$ ) was significantly higher than for those diagnosed by PBMC-PCR $(73 / 785,9.3 \% ; p<0.00001)$.
Table 2 demonstrates results of PBMC-PCR in diagnosing SNOCIs and COCIs. Prevalence of SNOCIs who presented with solitary positive anti-HCV IgG antibody in non-viremia patients $(34.82 \%)$ was highly significant (higher) compared to prevalence of COCIs (3.125\%) in negative controls for both IgG antibodies and viremia $(p<0.00001)$. Thus, SNOCIs are highly recommended for diagnostic testing and meticulous evaluation before proposal of HCV eradication.

\section{SCSD of RNA-genomic materials}

Tables 1 and 3 illustrate evaluation of SCSD as detected by SRT-PCR and PBMC-PCR in anti-HCV IgG antibody-positive naïve patients. The overall prevalence of SCSD dropped from $14.26 \%$ by SRT-PCR to $9.3 \%$ by PBMC-PCR ( $p=$ 0.003 ), as shown in Table 1 . Correlation of SCSD with hepatic cirrhosis in naïve cirrhotic cases, non-cirrhotic patients and controls is demonstrated in Table 3. SCSD of RNA genomic materials was significantly lower in post-HCV non-viremia patients regardless of cirrhosis compared to controls, as diagnosed by both SRT-PCR and PBMC-PCR $(p<$ $0.00001)$. Upon comparing the diagnostic yields of SRT-PCR with PBMC-PCR regarding evaluation of SCSD, the latter procedure was associated with significantly lower SCSD of RNA ( $p=0.03$ ) because of higher sensitivity. Correlation of results of SRT-PCR and PBMC-PCR with hepatic cirrhosis is illustrated in Figure 2. SCSD of HCV RNA genomic materials, as diagnosed by SRT-PCR, had insignificant difference when comparing cirrhotic patients with non-cirrhotic naïve patients ( $p=0.446)$. On the other hand, PBMC-PCR had a highly significant difference in SCSD of HCV strands when comparing non-cirrhotic patients with cirrhotic naïve patients $(p=0.0001)$.

\section{Distribution of sense and antisense strands in cirrhotic versus non-cirrhotic naïve HCV infection}

As demonstrated in Table 4, naïve cirrhotic patients showed significantly increased prevalence of sense and antisense strands compared to naïve non-cirrhotic and negative controls $(p<0.001)$. The naïve non-cirrhotic patients, who presented with solitary positive serum anti-HCV IgG antibodies, had a significantly increased frequency of both sense and antisense strands compared to controls $(p<0.00001)$. On the other hand, Figure 3 shows the relationship between density of hepatic cirrhosis and the intracellular HCV RNA strands' distribution in naïve patients. Association of intraPBMC HCV genomic materials with hepatic fibrosis was

Table 3. Comparison of PBMC-PCR with SRT-PCR in evaluation of spontaneous HCV RNA disappearance in cirrhotic and non-cirrhotic naïve patients

\begin{tabular}{lll}
\hline HCV IgG Abs and PCR based grouping $(n)$ & Cirrhotic naïve n (\%) & Non-cirrhotic Naïve n (\%) \\
\hline IgG abs-negative/PCR negative Controls (62) & $0.0(0.0 \%)$ & $62(100 \%)$ \\
IgG abs-positive/SRT-PCR negative Naïve (112) & $57(50.89 \%)$ & $55(49.11 \%)$ \\
IgG abs-positive/PBMC-PCR negative Naïve (73) & $25(34.25 \%)$ & $48(65.75 \%)$ \\
Fisher's exact 2-tailed p: A vs. B & 0.00000000 \\
: A vs. C & 0.00000001 \\
: B vs. C & $\mathbf{0 . 0 3 3 8 4 3 5 2}$ & \\
\hline
\end{tabular}

Spontaneous disappearance of HCV RNA genomic materials from PBMCs is significantly lower than in serum from post-HCV naïve patients regardless of cirrhotic changes $(p=0.03)$. All controls are non-cirrhotic and showed significant absence of both serum and intra-PBMC HCV RNA genomic infection than all naïve post-HCV infection $(p>0.00001)$. 


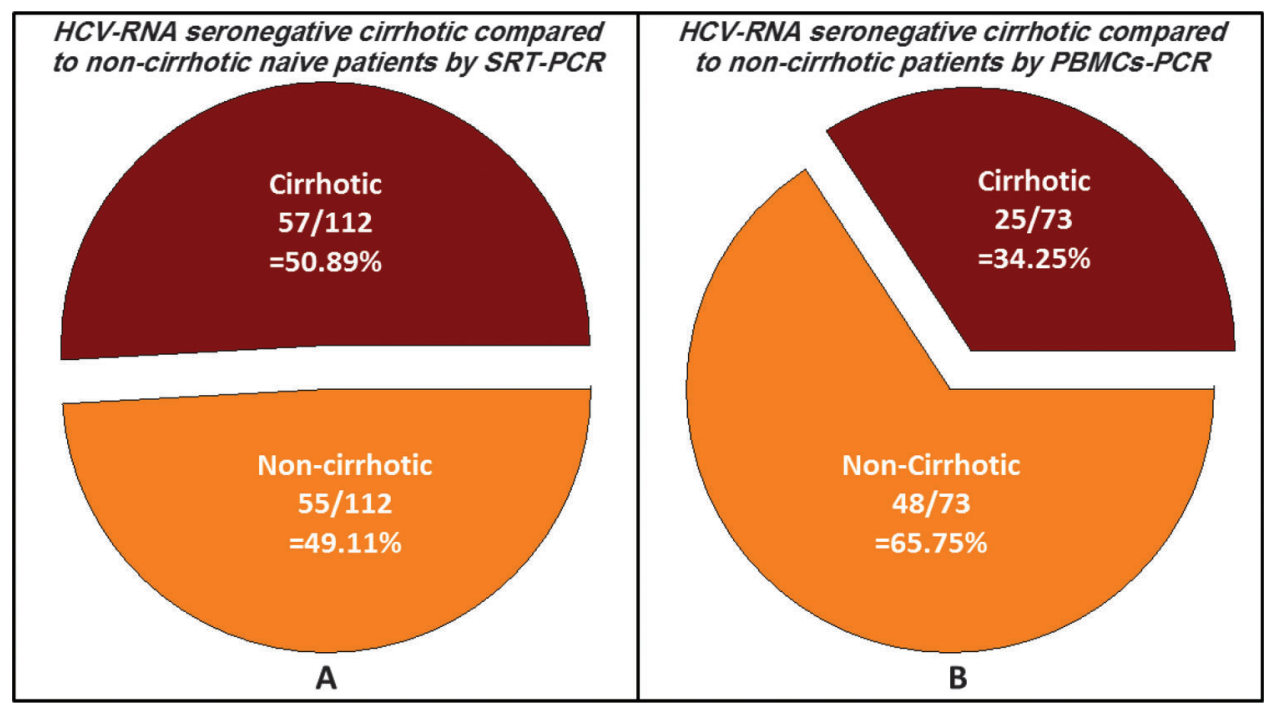

Fig. 2. SCSD of HCV RNA genomic materials from IgG antibody-positive and PCR negative populations in relation to hepatic cirrhosis. SCSD of HCV RNA as diagnosed by A) SRT-PCR and B) PBMC-PCR. A) Insignificant difference was found when cirrhotic and non-cirrhotic naïve patients were compared (Fisher's exact 1-tailed, $p=0.446)$. B) Significant difference was found when non-cirrhotic and cirrhotic naïve patients were compared (Fisher's exact 1 -tailed, $p=0.0001$ ).

Table 4. Distribution of sense and antisense HCV RNA strands in cirrhotic and non-cirrhotic naïve patients

\begin{tabular}{|c|c|c|c|}
\hline \multirow[b]{2}{*}{ Study subjects $(n)$} & \multicolumn{3}{|l|}{ PBMC-PCR } \\
\hline & Antisense strand $n$ ( $\%$ positive) & Sense strand $n$ ( $\%$ positive) & Negative $n$ (\% positive) \\
\hline Controls (62) & $0.0(0.00)$ & $2(3.2300)$ & $60(96.77)$ \\
\hline Cirrhotic naïve (57) & $11(19.30)$ & $21(36.84)$ & $25(43.86)$ \\
\hline Non-cirrhotic naïve (55) & $3(5.45)$ & $4(7.270)$ & $48(87.28)$ \\
\hline $\begin{array}{r}\text { Fisher's exact 2-tailed } p: \text { A vs. B } \\
\text { : A vs. C } \\
: \text { B vs. C }\end{array}$ & $\begin{array}{l}0.00000000 \\
0.00000000 \\
0.00000010\end{array}$ & $\begin{array}{l}0.000000028 \\
0.000000000 \\
0.000371340\end{array}$ & $\begin{array}{l}0.0034618535 \\
0.0000000000 \\
0.0026551955\end{array}$ \\
\hline
\end{tabular}

Naïve cirrhotic patients have significantly higher frequencies of both sense and antisense strands compared to naïve non-cirrhotic and negative controls ( $p<0.001$ ). Naïve non-cirrhotic patients have a significantly high frequency of sense and antisense strands compared to controls $(p<0.00001)$.

found to be highly significant in those who have intracellular RNA strand infection in comparison to the post-hepatitis PCR negative patients $(p<0.00001$ and 0.003 for intra-PBMC sense and antisense strands, respectively).

Hepatic fibrosis showed insignificant changes in distribution among the naïve HCV patients who had RNA-antisense or sense strand infections $(p=0.68)$. Quantitatively, the difference in hepatic fibrosis as $\mathrm{kPa}$ was very highly significant when HCV PCR negative, antisense and sense strand-infected patients were compared with negative controls $(p<$ $0.00001)$. Statistical analysis of absolute $\mathrm{kPa}$ values was done by using the mean values of hepatic fibrosis \pm standard deviation (SD) of the illustrated groups in Figure 3. The increased means of hepatic fibrosis were significantly associated with intra-cellular infections with sense $(19.17 \pm 14.28)$ and antisense (20.8 \pm 16.29$)$ strands compared to controls (3.34 \pm 0.34$)$ and post-HCV PCR negative patients (7.51 \pm 8.28; T-value $>1.0$ ). The mean values of hepatic fibrosis were insignificantly different for those who had intra-PBMC infection with HCV-RNA antisense strand (20.8 \pm 16.29$)$ upon comparison with those who had infection with sense strands $(19.17 \pm 14.28 ; T=0.74)$, and on comparison of
post-HCV PCR negative groups $(7.51 \pm 8.28)$ with negative controls ( $3.34 \pm 0.34 ; T=0.0001)$. These results confirm the statistically analyzed data that were retrieved from Figure 3.

\section{HCV RNA seroconversion compared to spontaneous disappearance of intracellular infection}

The fate of intra-PBMC HCV-RNA infection in 7 patients determined at 1-year follow-up is described in Table 5. At baseline, all 7 naïve patients were cirrhotic and had positive serum anti-HCV IgG antibodies, with 4 having both HCV strands inside their PBMCs and the other 3 having the intracellular RNA sense strand but lacking the antisense one. Follow-up of the 7 patients for 1 year by SRT-PCR and PBMC-PCR revealed the following: a) 3 out of 4 F4 patients $(75 \%)$ who had positive intra-PBMC sense and antisense strands at baseline showed RNA seroconversion ( $p=$ 0.047), with the fourth patient was still positive for intracellular sense and antisense RNA strands without RNA seroconversion; b) absence of antisense strand in the other 3 naïve patients who started with positive intra-PBMC HCV-RNA sense 


\section{Correlation of intra-PBMCs HCV genomic infection with hepatic fibosis in RNA seronegative patients}

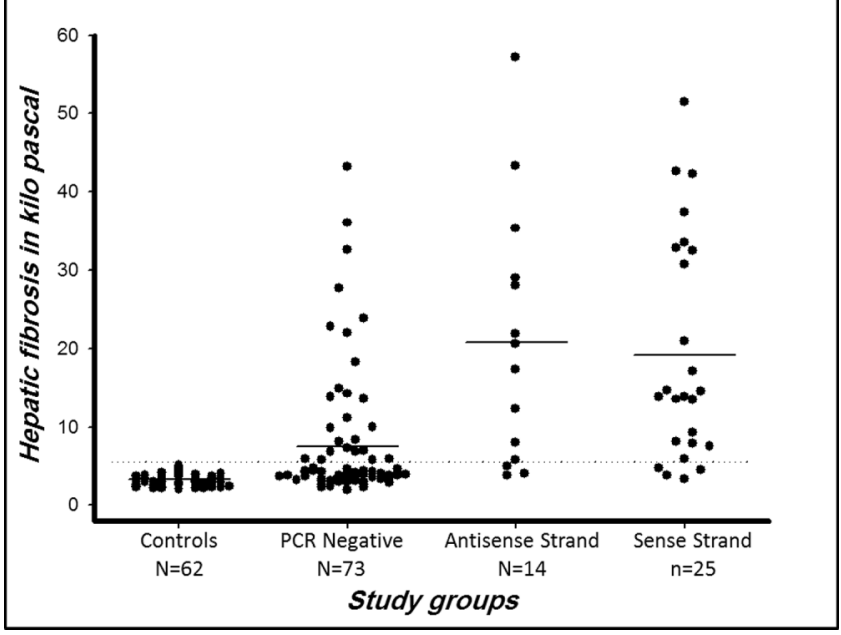

Fig. 3. Relative quantification of hepatic fibrosis per RNA seronegative subject in relation to intra-PBMC HCV-infection. Intracellular RNA was significantly associated with liver fibrosis upon comparison of currently infected groups with the post-HCV PCR negative group (Fisher's exact 2-tailed, $p=$ 0.00001 and $p=0.0028$, respectively, for intra-PBMC sense and antisense strands). Hepatic fibrosis was almost equally distributed among patients who presented with intra-PBMC HCV RNA antisense or sense strand infection (Fisher's exact 1 -tailed, $p=0.68$ ). Fisher's exact 1 -tailed $p<0.00001$ was found upon comparing controls with each of the other three groups. Dotted line, cutoff point; solid line, mean $\mathrm{kPa}$ values per group. Data cutoff point $=$ mean+3SD of controls $\mathrm{KPa}$ values.

strand were associated with clearance of the intracellular infection after 1 year.

\section{Discussion}

The current study presents the advantage of using PBMC-PCR in diagnosing OCIs. PBMC-PCR added $3.125 \%$ of asymptomatic COCIs and $34.82 \%$ of SNOCIs, significantly raising the overall number of infected cases from 673 to 712 . Infections that are usually recognized in clinical practice as cases with history of HCV infection (i.e. having no viremia despite positive serum IgG-antibodies) were designated as patients with SCSD in the current study. The number of cases presented with SCSD was found to be significantly lower because of the increased sensitivity of PBMC-PCR in diagnosing SNOCIs. The association of hepatic fibrosis with SNOCIs was also significant in the current data set. Furthermore, the increased density of hepatic fibrosis is accompanied by intraPBMC infection with either sense or antisense strands. The HCV RNA seroconversion in this cohort is attributed to intraPBMC infection with both sense and antisense strands in cirrhotic patients.

The increased sensitivity of diagnosing dormant HCVinfection addressed by a previous study ${ }^{5}$ adds major advantages to the management of both SNOCIs and SEOCIs in medical practice. The appreciated high predictive values of HCV-PCRs provide a trustworthy diagnostic tool to trace extra-and intra-cellular RNA replication. The current study looked for RNA strands in PBMCs because of their considerable degrees of HCV tropism and because their nuclei are essential to viral RNA genomic replication. ${ }^{6}$ The solitary intracellular recognition of HCV RNA, without viremia, has been known to occur during the incubation periods or as a post-infection presentation after successful elimination of genomic materials from sera. ${ }^{20}$

Spontaneous and drug-induced HCV RNA disappearance from sera have been collectively termed as sustained virologic disappearance; unfortunately, that naming convention doesn't correspond to elimination of viral genomic materials from the rest of body tissues. ${ }^{21}$ RNA seroconversion in both post-treatment or naïve patients is usually related to persistent intracellular infection with $\mathrm{HCV}$ genomic strands. ${ }^{9,11,22-25}$ We have recently evaluated PBMC-PCR of HCV-RNA as one of the most sensitive and specific tools to assess extrahepatic HCV infections. ${ }^{5}$ In current cohort, we utilized PBMC-PCR to identify HCV infections in naïve cases that tested negative by SRT-PCR (COCIs and SNOCIs). The significant increases in the numbers of the infected cases

Table 5. RNA seroconversion versus spontaneous intracellular disappearance of naïve HCV infection as recognized by concomitant SRT-PCR and PBMC-PCR

\begin{tabular}{|c|c|c|c|c|c|c|c|}
\hline \multirow[b]{3}{*}{$\begin{array}{l}\text { Serial } \\
\text { number }\end{array}$} & \multicolumn{3}{|c|}{ Baseline PCR data at study entry } & \multirow[b]{3}{*}{$\begin{array}{l}\text { Fibroscan results } \\
\text { at baseline in } \mathrm{kPa}\end{array}$} & \multicolumn{3}{|c|}{ Follow-up by PCR after one year } \\
\hline & \multirow[b]{2}{*}{$\begin{array}{l}\text { SRT-PCR } \\
\text { Serum RNA }\end{array}$} & \multicolumn{2}{|l|}{ PBMC-PCR } & & \multirow{2}{*}{$\begin{array}{l}\text { SRT-PCR } \\
\text { Serum RNA } \\
(\mathrm{IU} / \mathrm{ml})\end{array}$} & \multicolumn{2}{|l|}{ PBMC-PCR } \\
\hline & & Sense strand & Antisense strand & & & Sense strand & $\begin{array}{l}\text { Antisense } \\
\text { strand }\end{array}$ \\
\hline 1 & - ve & +ve & +ve & $\mathrm{F} 4=51.4$ & 21700 & +ve & +ve \\
\hline 2 & - ve & +ve & +ve & $\mathrm{F} 4=57.3$ & 242000 & +ve & +ve \\
\hline 3 & $-\mathrm{ve}$ & +ve & +ve & $\mathrm{F} 4=28.0$ & 299000 & +ve & +ve \\
\hline 4 & - ve & +ve & +ve & $\mathrm{F} 4=35.3$ & $-v e$ & +ve & +ve \\
\hline 5 & - ve & +ve & -ve & $\mathrm{F} 4=17.1$ & -ve & -ve & -ve \\
\hline 6 & -ve & +ve & -ve & $F 2=9.50$ & -ve & $-v e$ & -ve \\
\hline 7 & -ve & +ve & $-v e$ & $F 1=5.80$ & -ve & $-v e$ & -ve \\
\hline
\end{tabular}

Positive cases for both intra-PBMC HCV RNA strands at baseline are associated with RNA seroconversion in 3 out of 4 (uncorrected 2-tailed, $p=0.047$ ) F4 naïve patients; the remaining patient failed to clear the intracellular sense and antisense RNA strands. All naïve patients who had positivity for intra-PBMC HCV-RNA sense but negativity for antisense strand cleared the intracellular infection within 1 year. $\mathrm{KPa}$, Kilo Pascal; + ve, positive; $-\mathrm{ve}$, negative. F0 <5.5 kPa, F1 = 5.5-7.5 kPa, F2 = 7.5-10 kPa, F3 = 10-15 $\mathrm{kPa}, \mathrm{F} 4>15.0 \mathrm{kPa}$. 
after adding the newly diagnosed cases by PBMC-PCR recommends cellular screening for HCV-RNA infection to diagnose SNOCIs. On the other hand, the present results showed that COCIs prevalence is almost the same as described in other reports. $^{2-4}$

It is important to remember that the studied population has a higher incidence of HCV genotype 4 and a lower incidence of genotype 1 , a profile that is spreading widely in western countries. However, the current study did not aim to investigate OCIs for any particular genotype, but instead aimed to provide insight into a new use of an established diagnostic tool that would facilitate the diagnostic workup and furnish more accurate information regardless to viral genotype. The clinical application of the data analysis method that was adopted in the current study would be valid for studying of other populations and a spectrum of genotypes. However, calculation of the real prevalence of HCV infection in any population worldwide is dependent upon the combination of multiple diagnostic procedures (i.e. enzyme-linked immunosorbent assay, traditional PCR, PBMC-PCR, tissue PCR, ultracentrifugation-based PCR approach, etc.), 5,20 a countless number of tissues (i.e. serum, blood cells, whole blood, bone marrow, liver tissues, etc.), ${ }^{20,24,26}$ and application in various clinical case situations (i.e. treatment-naïve, treatment-experienced, in contact with $\mathrm{HCV}$ patients, and with chronic active hepatocellular damage). ${ }^{5,23-28}$

Our study concluded that SCSD is inversely proportional to the degree of hepatic cirrhosis; in addition, the increased hepatic cirrhosis is associated with diminished frequency of SCSD. These results confirm other studies regarding the association of liver cirrhosis with active HCV infection in viremic patients, ${ }^{10}$ but is considered a novel finding in SNOCIs as we reported in a previous study. ${ }^{5}$ It is convenient to consider the presence of HCV IgG antibody in naïve nonviremia (SCSD) patients as a remark for history of HCV infection, despite the belonging of dormant HCV infection in SCSD patients to the SNOCIs, as documented in the current study. Considering the clinical importance of PBMC-PCR, the significantly higher number of SCSD cases (73/112) compared to SNOCIs (39/112) supports the importance of anti-HCV IgG antibody testing as a preliminary screening approach, although it is not sufficient as a baseline diagnostic test for treatment. The numbers of SCSD cases are expected to drop down upon searching nucleated cells from other tissues (e.g., bone marrow, lymph nodes, spleen, liver, etc.) or using combined serologic diagnostic tests. ${ }^{20}$ The rising titers of antiHCV IgG antibodies in naïve and post-treatment cases might be a good predictor for current chronic HCV-infection.

Diagnosis of hepatic fibrosis in non-viremia patients is a confusing clinical situation that might push both physician and patients to face unexpected morbidity and mortality outcomes over time. Results of intra-PBMC HCV-RNA detection in the current study concern the above-mentioned clinical situation. Fortunately, the current report addresses HCV as an inducing factor for liver cirrhosis in SNOCIs, which will cause impaired liver function. The impact of hepatic fibrosis on SCSD of infection is most probably dependent upon interactions between the host immune system and the underlying pathogenic etiology. The current data set linked density of hepatic fibrosis with the presence of intra-PBMC HCV-RNA sense and antisense strands. Cumulative data during the next few years might help in creation of effective guidelines for $\mathrm{HCV}$ therapy to address treatment feasibility of solitary intracellular chronic $\mathrm{HCV}$ infection problems in non-viremia patients. Drawbacks of neglecting progressive hepatic cirrhosis are shown by evidence of active liver cell damage, so that lack of appropriate and timely medical management is extremely hazardous. Narrowing the spectrum of the etiologic factors in anti-HCV IgG antibody-positive non-viremia patients by management of SNOCIs in cirrhotic patients should minimize the over-time morbidity and mortality rates among chronic HCV infections.

On the other hand, differential recognition of intra-PBMC sense and antisense strands by PCR in our study provided elective information regarding their relationship to HCV RNA seroconversion in naïve patients. The present results indicate that solitary identification of intra-PBMC HCV-RNA sense strand would not be enough to induce viral genomic material seroconversion in naïve patients. The co-existence of sense with antisense strands seems to be crucial for HCV-RNA genomic materials to reappear in patient's sera. The authors believe that this is the first-report of HCV-RNA spontaneous seroconversion in naïve non-viremia cirrhotic patients occurring during the follow-up period without intervention, despite the small number ( 7 patients) that limits clinical significance. Contrary to these clues, the presence of intracellular antisense strand in naïve patients was reported as a good prognostic marker of response to antiviral therapy, and the post-treatment persistence of HCV-RNA antisense strands is an ominous sign because of the high probability of relapse. ${ }^{7}$

Findings of the current study include: a) diagnosis of both cryptogenic and SNOCIs by PBMC-PCR having significant impact upon the total prevalence of naïve HCV-infection; b) recognition of SNOCIs in all IgG antibody seropositive non-viremic patients being highly recommended because of their frequent detectability compared to controls; c) use of PBMC-PCR being strongly recommended to diagnose SCSD, in addition to SRT-PCR; d) cases of SCSD, as diagnosed by HCV PBMC-PCR, being more prevalent in non-cirrhotic patients than in cirrhotic patients; e) both intra-PBMC HCV-RNA sense and antisense strands being associated with increased density of hepatic fibrosis in kPa units; f) and primary seroconversion of HCV-RNA in naïve patients being associated with combined intra-PBMC existence of sense and antisense strands.

In conclusion, evaluation of intra-PBMC HCV RNA infection is crucial for diagnosing OCIs, evaluating post-infection sequelae in RNA seronegative naïve patients, and addressing the probability of viral RNA serologic relapse.

\section{Acknowledgments}

Financial support for this study was provided by the Faculty of Medicine at Al-Azhar University, National Research Center (Cairo, Egypt) and by the Academy of Scientific Research and Technology Development Fund (Grant No. 3365, to Mostafa K El-Awady). A grant from Alexion Corp. and the Herman Lopata Chair in Hepatitis Research is gratefully acknowledged.

\section{Conflict of interest}

The authors have no conflict of interests related to this publication.

\section{Author contributions}

Responsible for study design, patient management, data analysis, and writing of the manuscript (MDAA), recruited 
patients, revised and formatted the manuscript, and performed the virological investigation (SAE, GYW, MKEA).

\section{References}

[1] Pham TN, MacParland SA, Mulrooney PM, Cooksley H, Naoumov NV, Michalak TI. Hepatitis C virus persistence after spontaneous or treatment-induced resolution of hepatitis C. J Virol 2004;78:5867-5874. doi: 10.1128/JVI.78. 11.5867-5874.2004.

[2] Castillo I, Pardo M, Bartolomé J, Ortiz-Movilla N, Rodríguez-Iñigo E, de Lucas $S$, et al. Occult hepatitis $C$ virus infection in patients in whom the etiology of persistently abnormal results of liver-function tests is unknown. J Infect Dis 2004;189:7-14. doi: $10.1086 / 380202$.

[3] De Marco L, Gillio-Tos A, Fiano V, Ronco G, Krogh V, Palli D, et al. Occult HCV infection: an unexpected finding in a population unselected for hepatic disease. PLoS One 2009;4:e8128. doi: 10.1371/journal.pone.0008128.

[4] Bastani MN, Bokharaei-Salim F, Keyvani H, Esghaei M, Monavari SH, Ebrahimi $M$, et al. Prevalence of occult hepatitis $C$ virus infection in Iranian patients with beta thalassemia major. Arch Virol 2016;161:1899-1906. doi: 10. 1007/s00705-016-2862-3.

[5] Abd Alla MDA, El Awady MK. Hepatitis C virus RNA strands detection in peripheral blood mononuclear cells legitimizes virus eradication in negative serum PCR naïve and post-treatment patients. J Clin Transl Hepatol 2017;5: 1-8. doi: 10.14218/JCTH.2016.00054.

[6] Pawełczyk A, Kubisa N, Jabłońska J, Bukowska-Ośko I, Caraballo Cortes K, Fic $M$, et al. Detection of hepatitis C virus (HCV) negative strand RNA and NS3 protein in peripheral blood mononuclear cells (PBMC): CD3+, CD14+ and CD19+. Virol J 2013;10:346. doi: 10.1186/1743-422X-10-346.

[7] El-Awady MK, Ismail SM, El-Sagheer M, Sabour YA, Amr KS, Zaki EA. Assay for hepatitis $C$ virus in peripheral blood mononuclear cells enhances sensitivity of diagnosis and monitoring of HCV-associated hepatitis. Clin Chim Acta 1999;283:1-14. doi: 10.1016/S0009-8981(99)00007-8.

[8] De Marco L, Manzini P, Trevisan M, Gillio-Tos A, Danielle F, Balloco C, et al. Prevalence and follow-up of occult HCV infection in an Italian population free of clinically detectable infectious liver disease. PLoS One 2012;7:e43541. doi: 10.1371 /journal.pone.0043541.

[9] Keyvani H, Bokharaei-Salim F, Monavari SH, Esghaei M, Nassiri Toosi M, Fakhim $\mathrm{S}$, et al. Occult hepatitis $\mathrm{C}$ virus infection in candidates for liver transplant with cryptogenic cirrhosis. Hepat Mon 2013;13:e11290. doi: 10. 5812/hepatmon.11290.

[10] Attar BM, Van Thiel D. A new twist to a chronic HCV infection: occult hepatitis C. Gastroenterol Res Pract 2015;2015:579147. doi: 10.1155/2015/579147.

[11] Hajarizadeh B, Grebely J, Dore GJ. Epidemiology and natural history of HCV infection. Nat Rev Gastroenterol Hepatol 2013;10:553-562. doi: 10. 1038/nrgastro.2013.107.

[12] Hanno AFF, Mohiedeen KM, Alshayeb AF, Deghedy A. HCV RNA in peripheral blood mononuclear cells (PBMCs) as a predictor of the response to antiviral therapy in chronic hepatitis C. Alexandria Journal of Medicine 2014;50:317322. doi: 10.1016/j.ajme.2013.05.004.

[13] Colin C, Lanoir D, Touzet S, Meyaud-Kraemer L, Bailly F, Trepo C, et al. Sensitivity and specificity of third-generation hepatitis $C$ virus antibody detection assays: an analysis of the literature. J Viral Hepat 2001;8:87-95. doi: 10. 1046/j.1365-2893.2001.00280.x.
[14] Chomczynski P, Sacchi N. Single-step method of RNA isolation by acid guanidinium thiocyanate-phenol-chloroform extraction. Anal Biochem 1987; 162:156-159. doi: 10.1006/abio.1987.9999.

[15] Fong TL, Shindo M, Feinstone SM, Hoofnagle JH, Di Bisceglie AM. Detection of replicative intermediates of hepatitis C viral RNA in liver and serum of patients with chronic hepatitis C. J Clin Invest 1991;88:1058-1060. doi: 10.1172/JCI115368.

[16] Goergen B, Jakobs S, Symmons P, Hornes E, Meyer zum Büschenfelde KH, Gerken G. Quantitation of HCV-replication using one-step competitive reverse transcription-polymerase chain reaction and a solid phase, colorimetric detection method. J Hepatol 1994;21:678-682. doi: 10.1016/ S0168-8278(94)80118-5

[17] Löhr HF, Goergen B, Meyer zum Büschenfelde KH, Gerken G. HCV replication in mononuclear cells stimulates anti-HCV-secreting $B$ cells and reflects nonresponsiveness to interferon-alpha. J Med Virol 1995;46:314-320. doi: 10. 1002/jmv.1890460405.

[18] Bonnard P, Elsharkawy A, Zalata K, Delarocque-Astagneau E, Biard L, Le Fouler $L$, et al. Comparison of liver biopsy and noninvasive techniques for liver fibrosis assessment in patients infected with HCV-genotype 4 in Egypt. J Viral Hepat 2015;22:245-253. doi: 10.1111/jvh.12285.

[19] Al-Hamoudi W, Mohamed H, Abaalkhail F, Kamel Y, Al-Masri N, Allam N, et al. Treatment of genotype 4 hepatitis $C$ recurring after liver transplantation using a combination of pegylated interferon alfa-2a and ribavirin. Dig Dis Sci 2011;56:1848-1852. doi: 10.1007/s10620-010-1526-5.

[20] Castillo I, Bartolomé J, Quiroga JA, Barril G, Carreño V. Diagnosis of occult hepatitis C without the need for a liver biopsy. J Med Virol 2010;82 1554-1559. doi: 10.1002/jmv.21866.

[21] Jakobsen JC, Nielsen EE, Feinberg J, Katakam KK, Fobian K, Hauser G, et al. Direct-acting antivirals for chronic hepatitis C. Cochrane Database Syst Rev 2017;6:CD012143. doi: 10.1002/14651858.CD012143.pub2.

[22] Aboalam HA, Rashed HAG, Mekky MA, Nafeh HM, Osman OA. Prevalence of occult hepatitis $C$ virus in patients with $\mathrm{HCV}$-antibody positivity and serum HCV RNA negativity. J Curr Med Res Pract 2016;1:12-16. doi: 10.4103/ 2357-0121.192539.

[23] Baid-Agrawal S, Schindler R, Reinke P, Staedtler A, Rimpler S, Malik B, et al. Prevalence of occult hepatitis $C$ infection in chronic hemodialysis and kidney transplant patients. J Hepatol 2014;60:928-933. doi: 10.1016/j.jhep.2014. 01.012.

[24] Vidimliski PD, Nikolov I, Geshkovska NM, Dimovski A, Rostaing L, Sikole A. Review: Occult hepatitis $C$ virus infection: still remains a controversy. J Med Virol 2014;86:1491-1498. doi: 10.1002/jmv.23979.

[25] Elmasry S, Wadhwa S, Bang BR, Cook L, Chopra S, Kanel G, et al. Detection of occult hepatitis $C$ virus infection in patients who achieved a sustained virologic response to direct-acting antiviral agents for recurrent infection after liver transplantation. Gastroenterology 2017;152:550-553.e8. doi: 10.1053/j.gastro.2016.11.002.

[26] Rezaee-Zavareh MS, Hadi R, Karimi-Sari H, Hossein Khosravi M, Ajudani R, Dolatimehr $\mathrm{F}$, et al. Occult HCV infection: the current state of knowledge. Iran Red Crescent Med J 2015;17:e34181. doi: 10.5812/ircmj.34181.

[27] El Shazly Y, Hemida K, Rafik M, Al Swaff R, Ali-Eldin ZA, GadAllah S. Detection of occult hepatitis $C$ virus among healthy spouses of patients with HCV infection. J Med Virol 2015;87:424-427. doi: 10.1002/jmv.24074.

[28] Rezaee Zavareh MS, Alavian SM, Karimisari H, Shafiei M, Saiedi Hosseini SY. Occult hepatitis $C$ virus infection in patients with autoimmune hepatitis. Hepat Mon 2014;14:e16089. doi: 10.5812/hepatmon.16089. 